\title{
MIMO Systems with Intentional Timing Offset
}

\author{
Aniruddha Das (Nandan) ${ }^{1}$ and Bhaskar D. Rao ${ }^{2}$ \\ ${ }^{1}$ ViaSat Inc., Carlsbad, CA 92009, USA \\ ${ }^{2}$ Center for Wireless Communication at the University of California San Diego (UCSD), La Jolla, CA 92093, USA
}

Correspondence should be addressed to Aniruddha Das (Nandan), nandan@gmail.com

Received 3 November 2010; Revised 4 February 2011; Accepted 6 March 2011

Academic Editor: Athanasios Rontogiannis

Copyright ( 2011 A. Das (Nandan) and B. D. Rao. This is an open access article distributed under the Creative Commons Attribution License, which permits unrestricted use, distribution, and reproduction in any medium, provided the original work is properly cited.

The performance of MIMO systems with intentional timing offset between the transmitters has recently been the focus of study of different researchers. In these schemes, a nonzero (but known) symbol timing offset is introduced between the signals transmitted from the different transmitters to improve the performance of MIMO systems. This leads to a reduction in Interantenna Interference (IAI), and it is shown that an advanced receiver can utilize this information to extract significant performance gains. In this paper, we show that this transmission scheme may be used in conjunction with different kinds of receivers including ZF, MMSE, and sequence detection-based receivers. We also consider the design of novel pulse shapes that reduce the IAI at the expense of slightly higher intersymbol interference (ISI) and show that additional gains may be achieved.

\section{Introduction}

In multiple input multiple output (MIMO) communication systems, typically, the transmitters are all collocated, and the system is designed such that the symbol boundaries are aligned at the transmitters and also at the receivers (assuming no differential path delay). It has been shown in [1] that under the assumption of a richly scattered environment, such a system can lead to very high spectral efficiencies.

Practical communication systems typically use pulse shaping such as the square root raised cosine (SRRC) to limit the bandwidth occupied by the signal (see Chapter 9 of [2], [3], [4]). These pulses typically have an "excess bandwidth" which is usually denoted by a factor $0 \leq \beta \leq$ 1. The presence of excess bandwidth was used to improve performance in a fractionally sampled orthogonal frequency division multiplexing (OFDM) system in [5], where the cause of gain was similar to that discussed in this paper even though the system under consideration was very different.

We showed some preliminary results and demonstrated that significant gains could be obtained via a system with intentionally offset transmissions in [6]. Independently, and at about the same time, Shao et al. also presented a similar MIMO scheme with subsymbol timing offsets between the transmitted signals $[7,8]$, and Wang et al. presented a frequency domain equalization scheme for MIMO OFDM with intentional timing offsets in [9]. More recently, the capacity of MIMO systems with asynchronous pulse amplitude modulation (PAM) was studied in [10] where the authors show that this offset transmission scheme increases the capacity of such MIMO systems.

Delay diversity schemes for transmission, proposed previously (see, e.g., $[11,12]$ ), might appear to be similar to the proposed scheme, since those schemes also involve offset transmission. However, there are a couple of significant differences. First, delay diversity transmit schemes aim to increase the spatial diversity by transmitting the same (or precoded) data stream whereas in our proposed scheme, independent streams are transmitted from the different antennae preserving maximum spatial multiplexing gain. Second, in delay diversity schemes, the delays introduced are typically of a symbol duration or longer, whereas the intertransmitter timing offset here is of a subsymbol duration. Recent standards such as the Draft 802.11n as well as 3GPP LTE have included cyclic delay diversity (CDD), as a modification of delay diversity techniques proposed by [11]. These are typically applied in conjunction with an OFDM scheme, and so even though the delays could be a fraction of an OFDM symbol, these techniques are 
generally presented as a precoding scheme designed to increase the inherent diversity of the channel [13]. In our case, the intent of introducing the offset between the different transmit antennas in a single carrier system is to reduce the inter antenna interference (IAI) and introduce inter symbol interference (ISI) in the modulation while keeping maximum spatial multiplexing gain.

In MIMO systems, unlike in single-antenna systems, the multiple transmitters interfere with each other at each receive antenna resulting in IAI. In the absence of perfect Nyquist pulse shaping (or due to timing offset), ISI is introduced. Thus, there are two sources of impairment, ISI and IAI, that are distinct, and each one leads to a degradation in performance. In traditional aligned systems with Nyquist pulse shaping, there is little to no ISI, but on average, the IAI power is the same as that of the desired signal. In this paper, we show that by offsetting the transmit symbols relative to each other the IAI power can be reduced. In addition, we show that by using a different pulse shape that trades off ISI with the IAI, gains may be achieved practically for free. Although there is a large volume of prior research in the design of quasizero ISI practical pulse shapes that conform to various criterion such as spectral mask requirements, robustness to timing jitter, and peak-to-average power ratio (e.g., $[2,14-18]$ and references therein), to our knowledge, this is the first time that pulses have been designed with this criterion of lowering the IAI.

To summarize, the contributions of this paper are the following: we demonstrate the practical gains that may be achieved in a single carrier MIMO system by intentionally introducing a subsymbol delay offset between the transmitted waveforms. We show the performance of zero forcing (ZF), minimum mean squared error (MMSE) and sequence detection based receivers with SRRC pulse shapes and show that the performance is always better than that of the corresponding traditional MIMO system with timing aligned transmission, contrary to previously published research (for more details, please see Section 5). We also introduce a novel new pulse shape that lowers the energy at half symbol offsets, thus reducing the IAI and improving performance.

The remainder of this paper is organized in the following sections. In Section 3, we present an intuitive rational behind the superior performance of MIMO systems with timing offsets. Then, in Section 4, we present the analytical system model. In Section 5, different receiver structures are discussed. A novel pulse shape design criterion is given in Section 6 and following which simulation results are presented in Section 7 before concluding.

\section{Notation}

The notation adopted is as follows: lowercase boldface indicates a vector quantity, as in a. A matrix quantity is indicated by uppercase boldface as in A. Some of the most widely used symbols used throughout this paper are tabulated below. The rest of the variables will be defined as and when they appear throughout the paper (see Table 1).

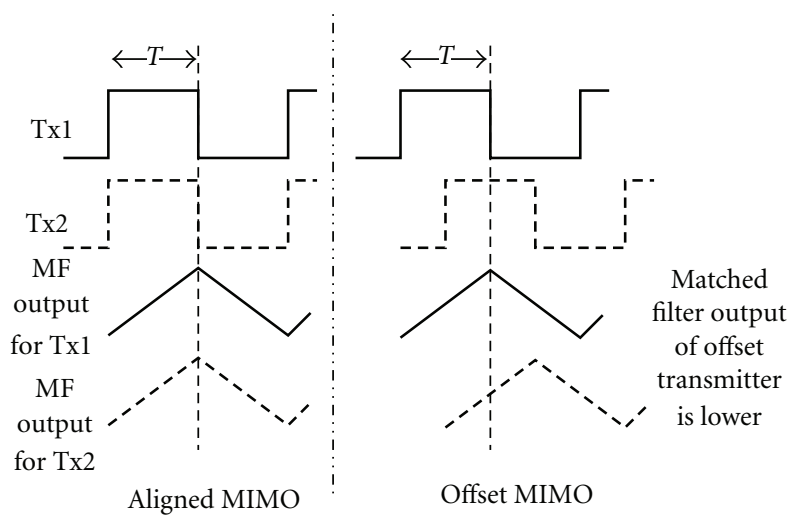

FIGURE 1: Reduction of interference power in offset MIMO.

\section{Motivation behind Timing Offset}

In this section, we present an intuitive rationale behind the improved performance of the offset MIMO system. In traditional single carrier MIMO systems, each receive chain downconverts the received signal to baseband, carries out analog to digital conversion, and then employs matched filtering before downsampling the received signal to the system symbol rate. Assuming equal channel gain, the signals from the symbol aligned transmitters contribute equal power to the received signal at the output of the downsampled received matched filter. It may be shown that in a rich scattering environment, the channel gains are statistically independent, and thus the receiver can demodulate the independent streams in either successive interference cancellation mode or joint detection mode.

In the offset scheme proposed, the transmitters' symbol boundaries are offset in time. Thus, when receiver-matched filtering is employed, under equal gain channel conditions the signals from the two transmitters are not of the same power. This is shown in Figure 1 for rectangular pulse shaping. Indeed, the received signal power from the transmitter with the offset symbol is lower than that from the transmitter which has its symbol boundaries aligned to that used by the received matched filter. Thus, for the same channel, the offset scheme has lower IAI power in comparison to that in the aligned case.

The amount of reduction in interference power depends on the pulse shape. While rectangular pulse shaping with half a symbol offset leads to a $3 \mathrm{~dB}$ reduction in interference power, most practical systems use bandlimited pulse shaping schemes using Nyquist pulse shapes such as the SRRC pulse shape. The interference reduction for various pulse shapes is obtained by sampling the convolution of the two pulses shapes (one at the transmitter and one at the receiver) at the various offsets. Since it is known that the convolution of two SRRC filters is the raised cosine filter, the IAI power 
TABLE 1

\begin{tabular}{lll}
\hline Symbol & Definition & Comments \\
\hline$\beta$ & Excess bandwidth of Nyquist pulses & Real scalar, $0 \leq \beta \leq 1$ \\
$T$ & Symbol duration & Real scalar \\
$\tau_{k}$ & Offset of symbol boundaries of Tx $k$ relative to Tx 0 & Real scalar, $0 \leq \tau_{k}<T$ \\
$M_{T}$ & Number of transmitters & Real scalar \\
$M_{R}$ & Number of receivers & Real scalar \\
$h_{i j}$ & Complex channel gain between $j$ th Tx and $i$ th Rx & Complex scalar \\
$\mathbf{H}_{\mathbf{k}}$ & Diagonal matrix whos $i$ ith entry is $h_{i k}$ & Complex, $M_{T} \times M_{T}$ matrix \\
$\mathbf{y}_{k}[i]$ & ith group of $M_{T}$ outputs at the $k$ th receiver & Complex, $M_{T} \times 1$ vector \\
$b_{k}[i]$ & ith transmitted symbol from $k$ th transmitter & Complex, scalar \\
$\mathbf{n}_{k}[i]$ & $i$ th noise vector at $k$ th receiver & Complex, $M_{T} \times 1$ vector \\
$E()$ & Expectation operator & n/a \\
()$^{H}$ & Hermitian operator & n/a \\
()$^{t}$ & Transpose operator & n/a \\
$\dagger$ & Pseudoinverse operator & n/a \\
$\mathbf{R}_{\mathbf{x y}}$ & $E\left[\mathbf{x y}{ }^{H}\right]$ & Cov matrix of zero mean vectors, $x$ and $y$ \\
\hline
\end{tabular}

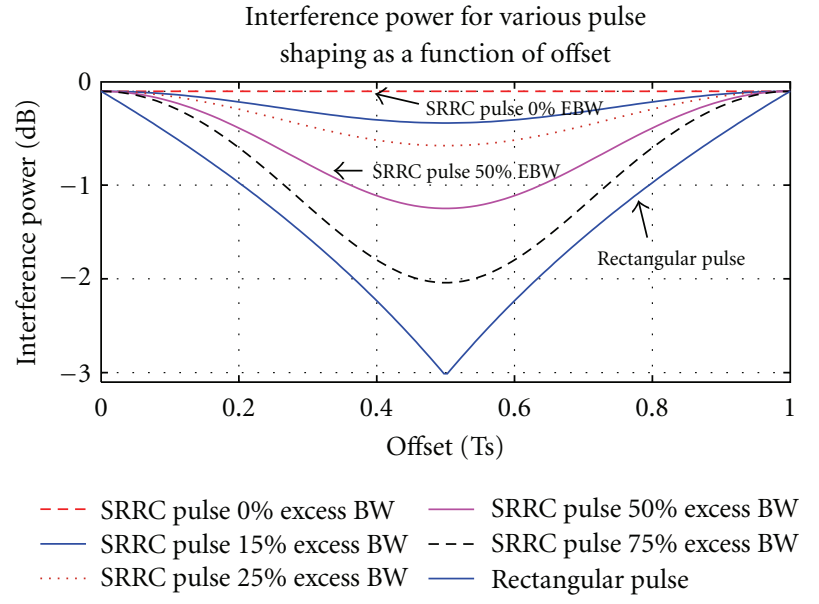

Figure 2: Interference power for various excess bandwidths and offsets. Note: no gain at 0 excess BW.

at an offset $\tau_{1}$ for a SRRC transmit pulse shape with excess bandwidth $\beta$ and symbol duration $T$, is given by

$$
\operatorname{IAI}\left(\tau_{1}\right)=\sum_{k=-\infty}^{k=\infty}\left(\frac{\sin \left(\pi\left(k T+\tau_{1}\right) / T\right)}{\pi\left(k T+\tau_{1}\right) / T} \frac{\cos \left(\pi \beta\left(k T+\tau_{1}\right) / T\right)}{1-\left(2 \beta\left(k T+\tau_{1}\right) / T\right)^{2}}\right)^{2}
$$

and is shown for various offsets and $\beta$ in Figure 2 below. The above formula samples the raised cosine pulse [19, equation (3)], at symbol intervals as a function of the offset from the symbol boundary, $\tau_{1}$, and determines the power thus obtained. It may be seen that for a pulse with no excess bandwidth $(\beta=0)$, there is no reduction in interference power, and thus no gains. However, as the excess bandwidth increases, the interference power reduces, and thus gains increase.

In addition to the lowering of interference power, the system performs better for one more reason. Offsetting the two transmit waveforms relative to each other introduces ISI, thus effectively converting the memoryless modulation schemes into those with memory. Consequently, an intelligent receiver can use the ISI to predictively cancel the interference in subsequent symbols, thus leading to an even greater suppression of interference

These two effects combine to provide significant system gains to a MIMO system with intentional timing offset in comparison to an equivalent symbol synchronous MIMO system.

\section{The Timing Offset MIMO System}

Figure 3 shows an offset of $\tau_{1}$ in a particular embodiment of the proposed system with 2 transmit antennas. The symbol duration is denoted by $T$ with $0 \leq \tau_{1}<T$. Other embodiments of the proposed system using $M_{T}$ antennas would have different $\tau_{k}$ s offsetting the signals from the different transmitters. For simplicity of illustration, the transmit signals are depicted with a rectangular pulse shape in Figure 3.

4.1. A $2 \times 2$ MIMO System with Timing Offset. For simplicity of presentation, a $2 \mathrm{Tx}-2 \mathrm{Rx}$ system with a rectangular pulse shaping is considered first. The signals transmitted from the 2nd transmitter is intentionally offset with respect to the first by $\tau_{1}$. Unlike in traditional symbol aligned MIMO, where the output of the matched filter downsampled to the symbol rate at the optimal sampling points are the sufficient statistics for estimating the transmitted symbols, in timing offset MIMO, the matched filter output of each receiver is sampled every $k T$ as well as every $k T+\tau_{1}$, where $k=0,1,2, \ldots$, thus collecting the output sampled optimally for both transmitters.

Let $h_{i j}$ be the complex path gain from the $j$ th transmitter to the $i$ th receiver. Then, stacking the $i$ th output of the two 


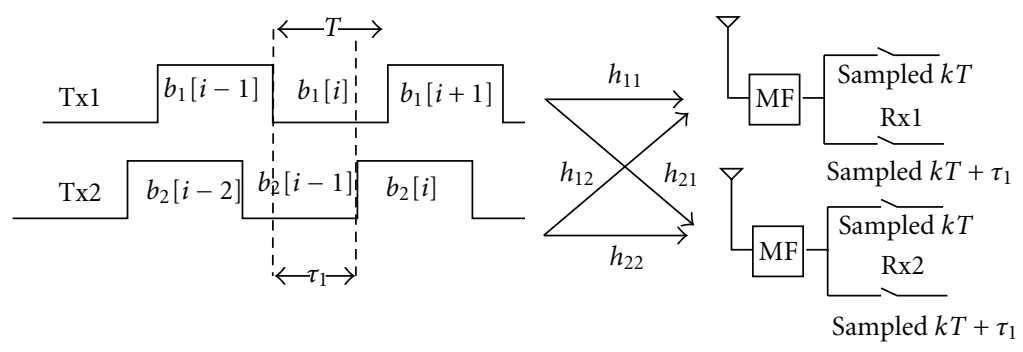

Figure 3: Subsymbol timing offset: 2 Tx antennas.

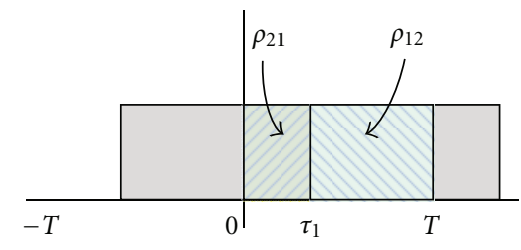

FIGURE 4: Cross correlations, $\rho_{12}$ and $\rho_{21}$.

matched filters, the received vector for each of the receive antennas is given by

$$
\begin{aligned}
\mathbf{y}_{1}[i]= & {\left[\begin{array}{cc}
0 & 0 \\
h_{11} \rho_{21} & 0
\end{array}\right]\left[\begin{array}{l}
b_{1}[i+1] \\
b_{2}[i+1]
\end{array}\right]+\left[\begin{array}{cc}
h_{11} & h_{12} \rho_{12} \\
h_{11} \rho_{12} & h_{12}
\end{array}\right] } \\
& \times\left[\begin{array}{l}
b_{1}[i] \\
b_{2}[i]
\end{array}\right]+\left[\begin{array}{cc}
0 & h_{12} \rho_{21} \\
0 & 0
\end{array}\right]\left[\begin{array}{l}
b_{1}[i-1] \\
b_{2}[i-1]
\end{array}\right]+\mathbf{n}_{1}[i], \\
\mathbf{y}_{2}[i]= & {\left[\begin{array}{cc}
0 & 0 \\
h_{21} \rho_{21} & 0
\end{array}\right]\left[\begin{array}{l}
b_{1}[i+1] \\
b_{2}[i+1]
\end{array}\right]+\left[\begin{array}{cc}
h_{21} & h_{22} \rho_{12} \\
h_{21} \rho_{12} & h_{22}
\end{array}\right] } \\
& \times\left[\begin{array}{l}
b_{1}[i] \\
b_{2}[i]
\end{array}\right]+\left[\begin{array}{cc}
0 & h_{22} \rho_{21} \\
0 & 0
\end{array}\right]\left[\begin{array}{l}
b_{1}[i-1] \\
b_{2}[i-1]
\end{array}\right]+\mathbf{n}_{2}[i],
\end{aligned}
$$

where $\mathbf{y}_{k}[i]$ is the $i$ th pair of outputs of the matched filter in the $k$ th receiver, $b_{k}[\mathrm{i}]$ is the $i$ th transmitted symbol from the $k$ th transmitter, and $\mathbf{n}_{k}[i]$ is the AWGN noise vector at the $k$ th receiver. The first row of (2) is the output of the matched filter matched to the first transmitter, and the second row is the output of the matched filter matched to the second transmitter.

The crosscorrelations $\rho_{12}$ and $\rho_{21}$ are a function of the pulse shape and timing offset, with the detailed form given by (9). For a rectangular pulse, $\rho_{12}$ and $\rho_{21}$ are shown in Figure 4.

It is seen that when the received matched filter is aligned to the first transmitter, the $i$ th symbol of the first transmitter not only interferes with the $i$ th symbol of the second transmitter (as would be the case in standard aligned MIMO architectures) but also interferes with the $(i-1)$ th symbol of the second transmitter. However, the interference power is reduced due to the offset of the transmit pulses from the two transmitters.
Some simple algebraic manipulations of (2) allow us to write the received samples of receiver $k$ as

$$
\begin{aligned}
\mathbf{y}_{k}[i]= & {\left[\begin{array}{cc}
0 & \rho_{21} \\
0 & 0
\end{array}\right]^{t}\left[\begin{array}{cc}
h_{k 1} & 0 \\
0 & h_{k 2}
\end{array}\right]\left[\begin{array}{l}
b_{1}[i+1] \\
b_{2}[i+1]
\end{array}\right] } \\
& +\left[\begin{array}{cc}
1 & \rho_{12} \\
\rho_{21} & 1
\end{array}\right]\left[\begin{array}{cc}
h_{k 1} & 0 \\
0 & h_{k 2}
\end{array}\right]\left[\begin{array}{l}
b_{1}[i] \\
b_{2}[i]
\end{array}\right] \\
& +\left[\begin{array}{cc}
0 & \rho_{21} \\
0 & 0
\end{array}\right]\left[\begin{array}{cc}
h_{k 1} & 0 \\
0 & h_{k 2}
\end{array}\right]\left[\begin{array}{l}
b_{1}[i-1] \\
b_{2}[i-1]
\end{array}\right]+\mathbf{n}[i] .
\end{aligned}
$$

It will be seen later that (3) is a special case of the more general formula derived for any arbitrary number of transmitters in (7). The above equations for $\mathbf{y}_{1}[i]$ and $\mathbf{y}_{2}[i]$ may be combined and written more compactly in the following matrix format:

$$
\mathbf{r}[i]=\left[\begin{array}{l}
\mathbf{y}_{1}[i] \\
\mathbf{y}_{2}[i]
\end{array}\right]=\mathbf{P b}[i+1]+\mathbf{Q b}[i]+\mathbf{R} \mathbf{b}[i-1]+\mathbf{n}[i] .
$$

To elucidate further, $\mathbf{P}, \mathbf{Q}$, and $\mathbf{R}$ are all $4 \times 2$ matrices, $\mathbf{b}[i]$ is a $2 \times 1$ vector and $\mathbf{n}[i]$ and $\mathbf{r}[i]$ are both $4 \times 1$ vectors.

When practical pulse shapes of longer duration such as the SRRC pulse shaping is used, then the interference from the offset is not limited to the adjacent symbols but depends on the length of the filter used. Although in theory the SRRC pulse is infinite in duration, all practical schemes use finite length pulse shapes. This may be seen in Figure 5, where a 10 -symbol long raised cosine pulse shape is shown. In this case, in an offset transmission scheme, the interference arises from 10 symbols as shown in Figure 5.

In this case, the expressions equivalent to (3) get more complex. Let $d(t)$ denote the continuous time convolution of the pulse shapes at the receiver and at the transmitter. $d(t)$ is assumed to be of duration $2 L$ and thus assumed to be zero for time, $t$, outside the interval $[-L T, L T]$. Let us define the two vectors

$$
\begin{aligned}
\mathbf{p}_{T} & =\left.d(t)\right|_{t=k T, k=-L \cdots L}=[d(-L T), \ldots d(0), \ldots d(L T)]^{t}, \\
\mathbf{p}_{\tau_{1}} & =\left.d(t)\right|_{t=k T+\tau_{1}, k=-L \cdots(L-1)} \\
& =\left[d\left(-L T+\tau_{1}\right), \ldots d\left(\tau_{1}\right), \ldots d\left((L-1) T+\tau_{1}\right)\right]^{t} .
\end{aligned}
$$




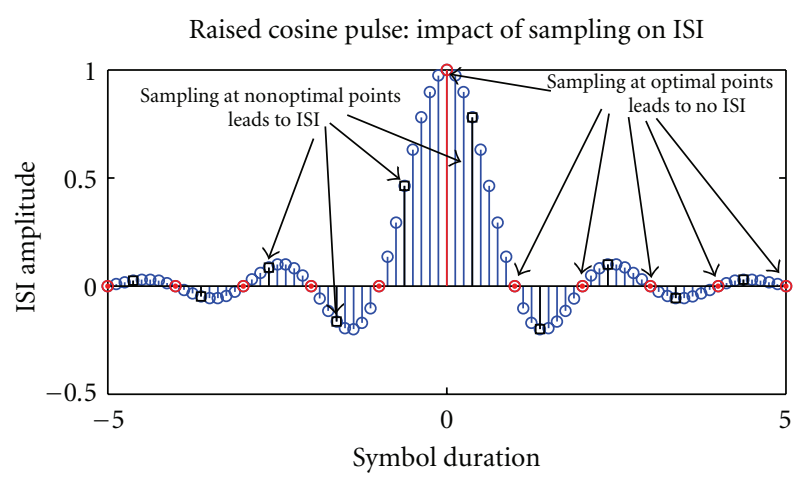

FIGURE 5: Raised cosine pulse: impact of sampling on ISI.

Thus, $\mathbf{p}_{T}$ consists of the samples of $d(t)$ at each of the symbol boundaries, and $\mathbf{p}_{\tau_{1}}$ consists of the samples of $d(t)$ at offsets of $\tau_{1}$ from the symbol boundaries. It is worth noting that if two infinitely long SRRC filters are convolved together to obtain $d(t)$, then $\mathbf{p}_{T}$ will consist of all zeros except for the middle element which will be 1 . In practice, however, this is usually not true and $\mathbf{p}_{T}$ will consider many nonzero elements, but usually, all are small relative to the middle element. As is the case for most practical pulse shapes, it is assumed that $d(t)$ is symmetric such that $d(-t)=d(t)$. Analogous to (3), the received samples at the $k$ th receiver matched to both the first and the second receiver may be expressed as

$$
\begin{aligned}
\mathbf{y}_{k}[i]= & \sum_{l=0}^{L}\left[\begin{array}{cc}
d(l T) & d\left(l T-\tau_{1}\right) \\
d\left(l T+\tau_{1}\right) & d(l T)
\end{array}\right]^{t}\left[\begin{array}{cc}
h_{k 1} & 0 \\
0 & h_{k 2}
\end{array}\right]\left[\begin{array}{l}
b_{1}[i+l] \\
b_{2}[i+l]
\end{array}\right] \\
& +\sum_{l=1}^{L}\left[\begin{array}{cc}
d(l T) & d\left(l T-\tau_{1}\right) \\
d\left(l T+\tau_{1}\right) & d(l T)
\end{array}\right]\left[\begin{array}{cc}
h_{k 1} & 0 \\
0 & h_{k 2}
\end{array}\right]\left[\begin{array}{l}
b_{1}[i-l] \\
b_{2}[i-l]
\end{array}\right] \\
& +\mathbf{n}_{k}[i] .
\end{aligned}
$$

4.2. $M_{T} \times M_{R}$ MIMO System with Timing Offset. The more general case with $M_{T}$ transmitters and $M_{R}$ receivers is now considered. In this setup, the relative timing offset between the first transmitter and $k$ th transmitter is $\tau_{k}$. Without loss of any generality, it is assumed that $0=\tau_{0} \leq \tau_{1} \leq \tau_{2} \cdots \leq$ $\tau_{M_{T}-1}<T$ where $T$ is the symbol duration. Each receiver conceptually has $M_{T}$ matched filters, each one matched to one of the transmitters (but in reality, would be implemented as a single matched filter sampled $M_{T}$ times a symbol). It should be mentioned that for excess bandwidth $0 \leq \beta \leq$ 1 , sampling each matched filter at 2 samples per symbol meets the Nyquist sampling criterion, and thus an intelligent receiver should be able to operate with the 2 samples/symbol out of the matched filter. In this analysis, we sample the output of the matched filer at $M_{T}$ samples per symbol only to keep the receiver structure conceptually simple.
For systems using pulse shapes $s_{l}(t)$ at the $l$ th transmitter such as the rectangular pulse that is zero outside $t \in[0, T]$, it may be shown that the samples received at the $k$ th receiver is a $M_{T} \times 1$ vector, $\mathbf{y}_{k}[i]$, that may be expressed as

$$
\mathbf{y}_{k}[i]=\left(\mathbf{R}_{\mathbf{1}}\right)^{t} \mathbf{H}_{k} \mathbf{b}[i+1]+\mathbf{R}_{\mathbf{0}} \mathbf{H}_{k} \mathbf{b}[i]+\mathbf{R}_{\mathbf{1}} \mathbf{H}_{k} \mathbf{b}[i-1]+\mathbf{n}_{k}[i],
$$

where the $M_{T} \times M_{T}$ matrix $\mathbf{H}_{k}=\operatorname{diag}\left(h_{k 1}, h_{k 2}, h_{k 3}, \ldots, h_{k M_{T}}\right)$ and the correlations $\rho_{k l}$ and $\rho_{l k}$ are given by:

$$
\begin{aligned}
& \rho_{k l}=\int_{\tau}^{T} s_{k}(t) s_{l}(t-\tau) d t, \\
& \rho_{l k}=\int_{0}^{\tau} s_{k}(t) s_{l}(t+T-\tau) d t .
\end{aligned}
$$

The entry in the $j$ th row, $k$ th column of the $M_{T} \times M_{T}$ matrices, $\mathbf{R}_{0}$ and $\mathbf{R}_{\mathbf{1}}$ is given by

$$
\begin{aligned}
& \mathbf{R}_{0}[j, k]= \begin{cases}1, & \text { if } j=k, \\
\rho_{j k}, & \text { if } j<k, \\
\rho_{k j}, & \text { if } j>k,\end{cases} \\
& \mathbf{R}_{\mathbf{1}}[j, k]= \begin{cases}0, & \text { if } j \geq k, \\
\rho_{k j}, & \text { if } j<k .\end{cases}
\end{aligned}
$$

It can be seen that (3) is a special case of (7) for $M_{T}=2$. The zero-mean Gaussian noise process $\mathbf{n}_{k}[i]$ has the following autocorrelation matrix, where $\sigma^{2}$ denotes the noise variance

$$
E\left[\mathbf{n}_{\mathbf{k}}[\mathbf{i}] \mathbf{n}_{\mathbf{1}}^{\mathrm{H}}[\mathbf{j}]\right]= \begin{cases}\sigma^{2}\left(\mathbf{R}_{\mathbf{1}}\right)^{t}, & \text { if } j=i+1, k=1 \\ \sigma^{2}\left(\mathbf{R}_{\mathbf{0}}\right)^{t}, & \text { if } j=i, k=l \\ \sigma^{2} \mathbf{R}_{\mathbf{1}}, & \text { if } j=i-1, k=1 \\ 0, & \text { otherwise. }\end{cases}
$$

It is noted that the expressions above are very similar to those in the derivation of the multiuser discrete time asynchronous model developed in [20, Section 2.10]. Although the notation has been chosen to be consistent with [20], the application space is quite different. We also note that comparing (7) with (14) of [8], it may be concluded that the received samples are identical in both our model, and in the case of offset MIMO presented by Shao et al. This was been shown by us in more detail in [21].

The derivations above can be extended for use with practical pulse shapes that extend beyond $t \in[0, T]$. Analogous to the derivation of (6), (7) can also be extended to the case where the convolution of the pulse shape at the transmit and the receive side $(d(t))$ is nonzero for $t \in$ $[-L T, L T]$ and is assumed to be zero for $t$ outside this interval. In that case, the received samples at the $k$ th receiver can be written as

$$
\mathbf{y}_{k}[i]=\sum_{l=0}^{L}\left(\mathbf{R}_{\mathbf{l}}\right)^{t} \mathbf{H}_{k} \mathbf{b}[i+l]+\sum_{l=1}^{L} \mathbf{R}_{\mathbf{l}} \mathbf{H}_{k} \mathbf{b}[i-l]+\mathbf{n}_{k}[i]
$$


where, like before, $\mathbf{H}_{k}$ is a $M_{T} \times M_{T}$ diagonal matrix given by $\mathbf{H}_{k}=\operatorname{diag}\left(h_{k 1}, h_{k 2}, h_{k 3}, \ldots, h_{k M_{T}}\right)$ and the $M_{T} \times M_{T}$ matrix, $\mathbf{R}_{\mathbf{l}}$ is given by

$$
\mathbf{R}_{\mathbf{l}}=\left[\begin{array}{cccccc}
d(l T) & d\left(l T-\tau_{1}\right) & d\left(l T-\tau_{2}\right) & \ldots & \ldots & d\left(l T-\tau_{M_{T}-1}\right) \\
d\left(l T+\tau_{1}\right) & d(l T) & d\left(l T-\left(\tau_{2}-\tau_{1}\right)\right) & 0 & \ldots & d\left(l T-\left(\tau_{M_{T}-1}-\tau_{1}\right)\right) \\
d\left(l T+\tau_{2}\right) & d\left(l T+\left(\tau_{2}-\tau_{1}\right)\right) & d(l T) & \ldots & \ldots & d\left(l T-\left(\tau_{M_{T}-1}-\tau_{2}\right)\right) \\
\ldots & \ldots & \ldots & \ldots & \ldots & \ldots \\
d\left(l T+\tau_{M_{T}-1}\right) & \ldots & \ldots & \ldots & \ldots & d(l T)
\end{array}\right] .
$$

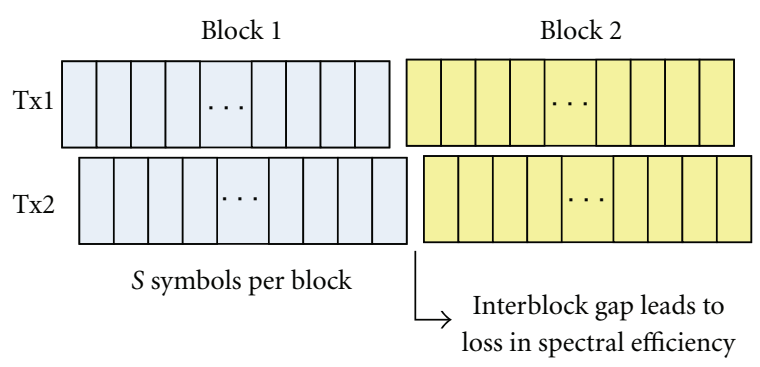

Figure 6: Block transmission scheme.

\section{Receiver Design}

In this section, we develop 3 different forms of receivers for the proposed system: (i) Zero Forcing (ZF) receivers, (ii) minimum mean squared error (MMSE) receivers and (iii) Viterbi algorithm-based sequence detection receivers.

All the receivers assume memoryless linear modulations such as $M$-ary Phase Shift Keying ( $M$-PSK) or $M$-ary quadrature amplitude modulation ( $M$-QAM) with a block transmission scheme as shown in Figure 6. It is assumed that there is no interblock interference (IBI). This condition can be satisfied by inserting an appropriate amount of idle time between the transmission of two blocks as shown in Figure 6. Each block is assumed to contain $S$ symbols long. Note that as $S$ increases, the overhead due to the interblock gap decreases. The transmitted symbols are assumed to be zero mean, unit energy, and uncorrelated in time and space. It is assumed that the channel is flat fading and unchanged over the duration of the entire block and independent from block to block and that the channel is known perfectly at the receiver. The noise is assumed to be Gaussian and independent of the data symbols. Two different noise models are used below-the first where the noise is spatially uncorrelated and the second where the noise has mutual coupling between the receivers.

5.1. ZF Receivers. In [8], the authors present a zero forcing (ZF) receiver whose performance is strongly dependent on the blocksize, $S$. They conclude that for large block sizes the performance of the offset transmission scheme is worse than that of the traditional MIMO schemes, and thus, the offset scheme should be used only for very short block sizes. In their work, the block sizes are typically 2,4 , or 10 symbols. This is a very severe restriction as such short block sizes lead to significant spectral efficiency reductions. With a block size of 2 symbols with 2 transmit antennas and offset $\tau_{1}=0.6 \mathrm{~T}$, the system has a spectral efficiency that is $23 \%$ less than that of synchronized systems and with a block size of 10 symbols, the spectral efficiency is reduced by $5.7 \%$. This reduction in spectral efficiency makes the offset MIMO scheme, proposed in [8], of limited use in practical systems.

A closer examination of the $\mathrm{ZF}$ receiver proposed by Shao et al. showed that it was not the optimal ZF receiver. This was first shown by us in [21]. The authors of [8] had mistakenly chosen a formulation that suffered a lot of noise enhancement as the block size, $S$, grew larger. To obtain the optimal ZF receiver, we first stack all the outputs of each block for the $k$ th receiver from (7) to obtain

$$
\mathbf{z}_{k}=\mathbf{R A}_{k} \mathbf{b}_{\text {block }}+\mathbf{n}_{k}
$$

where $\mathbf{z}_{k}=\left[\mathbf{y}_{k}^{t}(0), \mathbf{y}_{k}^{t}(1), \mathbf{y}_{k}^{t}(2), \ldots, \mathbf{y}_{k}^{t}(S-1)\right]^{t}$, the transmitted symbols, $\mathbf{b}_{\text {block }}=\left[\mathbf{b}^{t}(0), \mathbf{b}^{t}(1), \ldots, \mathbf{b}^{t}(S-1)\right]^{t}$ and $\mathbf{A}_{k}=$ $\operatorname{diag}\left\{\mathbf{H}_{k}, \mathbf{H}_{k}, \mathbf{H}_{k}, \ldots\right\} . \mathbf{y}_{k}(i)$ and $\mathbf{b}(i)$, both $M_{T} \times 1$ vectors, represent the received samples matched to each transmitter received at receiver $k$ at time $i$ and the transmitted symbols from all transmitters at time $i$, respectively. $\mathbf{H}_{\mathbf{k}}$ is a diagonal matrix of channel gains of size $M_{T} \times M_{T}$. Thus, in (13), $\mathbf{z}_{\mathbf{k}}$ is a $S M_{T} \times 1$ vector of all received samples in a block of $S$ transmitted symbols per transmit antenna at receiver $k$. $\mathbf{b}_{\text {block }}$ is the $S M_{T} \times 1$ vector of all transmitted symbols in that block, $A_{k}$ is a diagonal matrix of $S M_{T} \times S M_{T}$ elements of channel gains from the transmitters to the $k$ th receiver (assumed constant over the block). $\mathbf{R}$ is a $S M_{T} \times S M_{T}$ real symmetric correlation matrix given by (14), where $\mathbf{R}_{\mathbf{0}}$ and $\mathbf{R}_{\mathbf{1}}$ are given by (9)

$$
\mathbf{R}=\left[\begin{array}{cccccc}
\mathbf{R}_{\mathbf{0}} & \mathbf{R}_{\mathbf{1}}{ }^{t} & 0 & \ldots & \cdots & 0 \\
\mathbf{R}_{\mathbf{1}} & \mathbf{R}_{\mathbf{0}} & \mathbf{R}_{\mathbf{1}}{ }^{t} & 0 & \cdots & 0 \\
0 & \mathbf{R}_{\mathbf{1}} & \mathbf{R}_{\mathbf{0}} & \mathbf{R}_{\mathbf{1}}{ }^{t} & 0 & \ldots \\
\ldots & \ldots & \ldots & \ldots & \ldots & \ldots \\
0 & \cdots & 0 & \mathbf{R}_{\mathbf{1}} & \mathbf{R}_{\mathbf{0}} & \mathbf{R}_{\mathbf{1}}{ }^{t} \\
0 & \cdots & \ldots & 0 & \mathbf{R}_{\mathbf{1}} & \mathbf{R}_{\mathbf{0}}
\end{array}\right] .
$$


Then all the $\mathbf{z}_{\mathbf{k}}$ outputs of each receiver is stacked in the following manner:

$$
\begin{gathered}
{\left[\begin{array}{c}
\mathbf{z}_{1} \\
\mathbf{z}_{\mathbf{2}} \\
\vdots \\
\mathbf{z}_{M_{R}}
\end{array}\right]=\left[\begin{array}{cccc}
\mathbf{R} & 0 & \cdots & 0 \\
0 & \mathbf{R} & 0 & \cdots \\
\cdots & \cdots & \cdots & \cdots \\
0 & \cdots & 0 & \mathbf{R}
\end{array}\right]\left[\begin{array}{c}
\mathbf{A}_{1} \\
\mathbf{A}_{2} \\
\vdots \\
\mathbf{A}_{M_{R}}
\end{array}\right] \mathbf{b}_{\text {block }}+\left[\begin{array}{c}
\mathbf{n}_{1} \\
\mathbf{n}_{2} \\
\vdots \\
\mathbf{n}_{M_{R}}
\end{array}\right],} \\
\mathbf{z}_{\text {tot }}=\mathbf{R}_{\text {tot }} \mathbf{A}_{\text {tot }} \mathbf{b}_{\text {block }}+\mathbf{n}_{\text {tot }}
\end{gathered}
$$

and the optimum ZF receiver is given by

$$
\hat{\mathbf{b}}_{\mathrm{ZF} \mathrm{opt}}=\left(\mathbf{A}_{\mathrm{tot}}^{H} \mathbf{R}_{\mathrm{tot}} \mathbf{A}_{\mathrm{tot}}\right)^{-1} \mathbf{A}_{\mathrm{tot}}^{H} \mathbf{z}_{\mathrm{tot}} .
$$

The above optimal ZF receiver not only cancels all the interference, but it minimizes the output noise variance. It can be readily derived by noting that the optimal ZF receiver is the well known best linear unbiased estimator (BLUE) [22, Chapter 6]. This can be seen by noting that in the BLUE estimation, we seek an unbiased estimator which minimizes the estimator variances. The unbiased criterion ensures cancellation of interference while minimizing variance corresponds to maximizing signal to noise ratio.

It should be pointed out that the optimal ZF receiver is a batch receiver; that is, it works on the received samples from the entire block at the same time. This increases complexity and introduces latency in the system (since the first transmitted symbols can only be decoded after the samples corresponding to the last transmitted symbol in the block have been received). The above receiver also needs to calculate the pseudoinverse of a $S M_{T} \times S M_{T}$ matrix. The block sizes of practical systems often consists of hundreds (sometimes thousands) of symbols, and thus the complexity of this step is nontrivial and indeed could be impractical with current hardware.

In Section 7.5, we plot the performance of the optimal ZF receiver developed here and compare the performance to that in [8]. As will be seen, the optimal ZF receiver does not suffer any significant performance degradation when the block size is increased.

5.2. MMSE Receivers. The linear MMSE receiver is known [2] to outperform the ZF receiver and is considered in this section. The LMMSE estimate of $\mathbf{b}$, given observation $\mathbf{r}$, is given by $\mathbf{R}_{\mathrm{br}} \mathbf{R}_{\mathrm{rr}}^{\dagger} \mathbf{r}$, where $\dagger$ indicates the pseudoinverse and $\mathbf{R}_{\mathbf{b r}}=E\left[\mathbf{b r}^{H}\right]$ and $\mathbf{R}_{\mathbf{r r}}=E\left[\mathbf{r r}^{H}\right]$ [22]. It is known that for Gaussian noise, the MMSE solution and the LMMSE solution are the same and so the terms are used interchangeably here.

Two classes of MMSE receivers are analyzed. The first class carries out joint detection of the symbols, while the second carries out layered interference cancellation. For both these receiver types, one-shot receivers (i.e., those that estimate $\mathbf{b}[i]$, given $\mathbf{r}[i]$ ) and windowed receivers (i.e., those that estimate $\mathbf{b}[i]$ given $\mathbf{r}[i-W], \ldots \mathbf{r}[i], \ldots \mathbf{r}[i+W]$, thus implying a window length of $2 W+1$ ) are developed. We will also develop an MMSE joint batch receiver, that is, one that estimates all the transmitted symbols of the block, using all the received samples in that block.

5.2.1. One-Shot LMMSE Receiver, $(W=0)$. In this scenario, the observations, $\mathbf{r}[i]$, are given by (4), and only one measurement vector is used to estimate the corresponding information carrying symbols. It is assumed that: (a) $\mathbf{b}[i] \mathrm{s}$ are zero mean, unit energy, and uncorrelated in time, (b) $h_{i j}$ s, the channel gains, are perfectly known at receiver and do not change over the duration of a block of data, and (c) the additive Gaussian noise is spatially uncorrelated and also uncorrelated with the information carrying signal. Under these assumptions, from (4), we have

$$
\begin{gathered}
\mathbf{R}_{\mathbf{r r}}=\mathbf{P P}^{\mathrm{H}}+\mathbf{Q} \mathbf{Q}^{\mathrm{H}}+\mathbf{R R}^{\mathrm{H}}+\mathbf{R}_{\mathrm{NN}}, \\
\mathbf{R}_{\mathbf{b}[\mathrm{i}] \mathbf{r}}=\mathbf{Q}^{H} .
\end{gathered}
$$

In the symbol aligned $2 \times 2$ model (traditional MIMO), $\mathbf{R}_{\mathrm{NN}}$, the noise covariance matrix, is often modeled as $2 \times 2$ identity matrix scaled with the noise variance $\sigma^{2}$. This simple model assumes that the noise variance, $\sigma^{2}$ is the same for both the receive antennae and that there is no noise coupling between the antennas. In offset MIMO, we have 2 sets of matched filters per receiver and so $\mathbf{R}_{\mathrm{NN}}$ is a $4 \times 4$ matrix. By observing that the continuous time AWGN noise is zero mean and independent between the two receivers and by noting that part of the integration period for each symbol is the same between the two matched filters in the same receiver, it may be shown that $\mathbf{R}_{\mathrm{NN}}$ for this noise model is no longer a scaled identity matrix, but is given by (18), where $\sigma^{2}$ is the noise variance and $\rho_{12}$ is given by $(9)$

$$
\mathbf{R}_{\mathrm{NN}}=\left[\begin{array}{cccc}
\sigma^{2} & \rho_{12} \sigma^{2} & 0 & 0 \\
\rho_{12} \sigma^{2} & \sigma^{2} & 0 & 0 \\
0 & 0 & \sigma^{2} & \rho_{12} \sigma^{2} \\
0 & 0 & \rho_{12} \sigma^{2} & \sigma^{2}
\end{array}\right] .
$$

In the more general case where the noise is not assumed to be independent between the two antenna, the noise covariance matrix in the traditional symbol aligned $2 \times 2$ system is given by

$$
\mathbf{R}_{\mathrm{NNaligned}}=\left[\begin{array}{ll}
\sigma_{11}^{2} & \sigma_{12}^{2} \\
\sigma_{21}^{2} & \sigma_{22}^{2}
\end{array}\right],
$$

where $\sigma_{11}^{2}$ and $\sigma_{22}^{2}$ are, respectively, the noise variances of the 1 st receive antenna and the 2 nd receive antenna. $\sigma_{12}^{2}$ and $\sigma_{21}^{2}$ are, respectively, the covariance of the noise on the first receive antenna with that of the 2 nd receive antenna and viceversa. In all these cases, the noise is assumed to be zero mean.

In this model for the noise, (18) can also be more generalized and is determined to be

$$
\mathbf{R}_{\mathbf{N N}}=\left[\begin{array}{cccc}
\sigma_{11}^{2} & \rho_{12} \sigma_{11}^{2} & \sigma_{12}^{2} & \rho_{12} \sigma_{12}^{2} \\
\rho_{12} \sigma_{11}^{2} & \sigma_{11}^{2} & \rho_{12} \sigma_{12}^{2} & \sigma_{12}^{2} \\
\sigma_{21}^{2} & \rho_{12} \sigma_{21}^{2} & \sigma_{22}^{2} & \rho_{12} \sigma_{22}^{2} \\
\rho_{12} \sigma_{21}^{2} & \sigma_{21}^{2} & \rho_{12} \sigma_{22}^{2} & \sigma_{22}^{2}
\end{array}\right] .
$$


Using (17) and (18) or (20), the transmitted symbols are thus estimated at the receiver to be

$$
\widehat{\mathbf{b}[i]}=\text { Quant }\left\{\mathbf{R}_{\mathbf{b}[\mathbf{i}] \mathbf{r}} \mathbf{R}_{\mathbf{r r}}^{\dagger}(\mathbf{r}[i])\right\}
$$

where $\mathbf{r}[i]$ is a vector of all observations being used for the estimate of $\mathbf{b}[i]$, and the Quant $\{\cdot\}$ function is used to make hard decisions on the processed samples.

5.2.2. Adjacent Symbol LMMSE Receiver, $W=1$. From the observation model, it is clear that because of correlation between adjacent measurements, an LMMSE receiver that estimates the information symbols using measurements that span more than one symbol duration can lead to improvements. In this section, the adjacent symbol LMMSE receiver that utilizes the three received vectors to decide $\mathbf{b}[i]$ will be considered. Using (4), the received vectors used to determine $\mathbf{b}[i]$ are

$$
\begin{aligned}
\mathbf{r}[i-1] & =\mathbf{P} \mathbf{b}[i]+\mathbf{Q} \mathbf{b}[i-1]+\mathbf{R} \mathbf{b}[i-2]+\mathbf{n}[i-1], \\
\mathbf{r}[i] & =\mathbf{P b}[i+1]+\mathbf{Q} \mathbf{b}[i]+\mathbf{R} \mathbf{b}[i-1]+\mathbf{n}[i] \\
\mathbf{r}[i+1] & =\mathbf{P} \mathbf{b}[i+2]+\mathbf{Q} \mathbf{b}[i+1]+\mathbf{R} \mathbf{b}[i]+\mathbf{n}[i+1]
\end{aligned}
$$

These three equations may be stacked and expressed more compactly as

$$
\begin{aligned}
\mathbf{y}[i]= & {\left[\begin{array}{l}
\mathbf{R} \\
0 \\
0
\end{array}\right] \mathbf{b}[i-2]+\left[\begin{array}{l}
\mathbf{Q} \\
\mathbf{R} \\
0
\end{array}\right] \mathbf{b}[i-1]+\left[\begin{array}{l}
\mathbf{P} \\
\mathbf{Q} \\
\mathbf{R}
\end{array}\right] \mathbf{b}[i] } \\
& +\left[\begin{array}{l}
0 \\
\mathbf{P} \\
\mathbf{Q}
\end{array}\right] \mathbf{b}[i+1]+\left[\begin{array}{l}
0 \\
0 \\
\mathbf{P}
\end{array}\right] \mathbf{b}[i+2]+\mathbf{n}_{3}[i] \\
= & \mathbf{M}_{1} \mathbf{b}[i-2]+\mathbf{M}_{2} \mathbf{b}[i-1]+\mathbf{M}_{3} \mathbf{b}[i]+\mathbf{M}_{4} \mathbf{b}[i+1] \\
& +\mathbf{M}_{5} \mathbf{b}[i+2]+\mathbf{n}_{3}[i] .
\end{aligned}
$$

Note that $\mathbf{y}[i]$ and $\mathbf{n}_{3}[i]$ are $12 \times 1$ vectors, each $\mathbf{M}_{i}$ is a $12 \times 2$ matrix, and $\mathbf{b}[i]$ is a $2 \times 1$ vector. Thus, the LMMSE receiver is given by

$$
\begin{aligned}
\widehat{\mathbf{b}[i]} & =\text { Quant }\left\{\mathbf{R}_{\mathbf{b}[\mathbf{i}] \mathbf{y}} \mathbf{R}_{\mathbf{y y}}^{\dagger}(\mathbf{y}[i])\right\} \\
& =\text { Quant }\left\{\mathbf{M}_{3}^{H}\left(\sum_{i=1}^{5} \mathbf{M}_{\mathbf{i}} \mathbf{M}_{\mathbf{i}}^{\mathbf{H}}+\mathbf{R}_{\mathbf{N N}}\right)^{\dagger}(\mathbf{y}[i])\right\} .
\end{aligned}
$$

In this context, the covariance matrix of the noise vector $\mathbf{n}_{3}[i]$ given by $\mathbf{R}_{N N}$ is a matrix with similar structure as in (18) or (20) except that it is a $12 \times 12$ matrix. This approach can be extended to more general receivers using a wider window of received samples to estimate the $i$ th transmitted symbol.
5.2.3. MMSE Joint Batch Receivers. The above two MMSE receivers estimated the transmitted symbol vectors one at a time; that is, $\mathbf{b}[0]$ is estimated, then $\mathbf{b}[1]$ is estimated and so on until all the transmitted symbols of the block are estimated. In this section, we present the joint batch MMSE receiver. This receiver estimates all the transmitted symbols of the block $\mathbf{b}_{\text {block }}$ based on all the received samples from that block, $\mathbf{z}_{\text {tot }}$ (see (15)).

Similar to the subsections above, the optimal estimate is derived below as

$$
\begin{aligned}
& \widehat{\mathbf{b}}_{\text {MMSE-block }} \\
& \quad=\text { Quant }\left[E\left[\mathbf{b}_{\text {block }} \mathbf{z}_{\text {tot }}{ }^{H}\right]\left(E\left[\mathbf{z}_{\text {tot }} \mathbf{z}_{\text {tot }}{ }^{H}\right]\right)^{\dagger} \mathbf{z}_{\text {tot }}\right] \\
& =\text { Quant }\left[\mathbf{A}_{\text {tot }}^{H} \mathbf{R}_{\text {tot }}^{H}\left(\mathbf{A}_{\text {tot }} \mathbf{R}_{\text {tot }} \mathbf{A}_{\text {tot }}^{H} \mathbf{R}_{\text {tot }}^{H}+\mathbf{R}_{\mathbf{n}_{\text {tot }} \mathbf{n}_{\text {tot }}}\right)^{\dagger} \mathbf{z}_{\text {tot }}\right] .
\end{aligned}
$$

As discussed in Section 5.1, these batch receivers are significantly more complicated to implement and require taking the inverse of matrices of size $S M_{T} \times S M_{T}$. They also add latency to the system and are included here for the sake of completion.

5.2.4. MMSE Receivers with Layered Detection and Interference Cancellation. The two receivers discussed above carry out joint decoding of symbols transmitted from the two transmitters. However, a vertical bell labs layered space time(V-BLAST-) type approach [1] where one transmitter is decoded (using a LMMSE receiver), and then the decoded symbols are used to carry out interference cancellation was also designed. As shown in [1, 23], the layered approach achieves superior performance in the traditional symbolaligned case, and here, it is expected that the layered detection will also improve performance in the proposed offset scheme.

It is well known (see, e.g., [1, 23, 24]) that optimal ordering of the decoding layers leads to performance improvements. As [1] has shown, decoding the layer with the highest SINR (or the lowest error variance) yields the optimal ordering.

Using (17), in the case of the one-shot ( $W=0$ ) offset MIMO system, the error covariance matrix may be expressed as

$$
\begin{aligned}
& E\left[(\mathbf{b}-\hat{\mathbf{b}})(\mathbf{b}-\hat{\mathbf{b}})^{H}\right] \\
& \quad=\mathbf{R}_{\mathbf{b b}}-\mathbf{R}_{\mathbf{b r}} \mathbf{R}_{\mathbf{r r}}^{\dagger} \mathbf{R}_{\mathbf{r b}} \\
& \quad=\mathbf{I}_{2 \times 2}-\mathbf{Q}^{\mathrm{H}}\left(\mathbf{P} \mathbf{P}^{\mathrm{H}}+\mathbf{Q} \mathbf{Q}^{\mathbf{H}}+\mathbf{R} \mathbf{R}^{\mathbf{H}}+\mathbf{R}_{\mathrm{NN}}\right)^{\dagger} \mathbf{Q} .
\end{aligned}
$$

Thus, the error variance of decoding the symbol from the first transmitter is given by the magnitude of the $(1,1)$ element and the error variance of decoding the symbol from the second transmitter is given by the magnitude of the $(2,2)$ element of the $2 \times 2$ error covariance matrix. The layer that has the lower error variance (and hence higher SINR) is decoded first. 


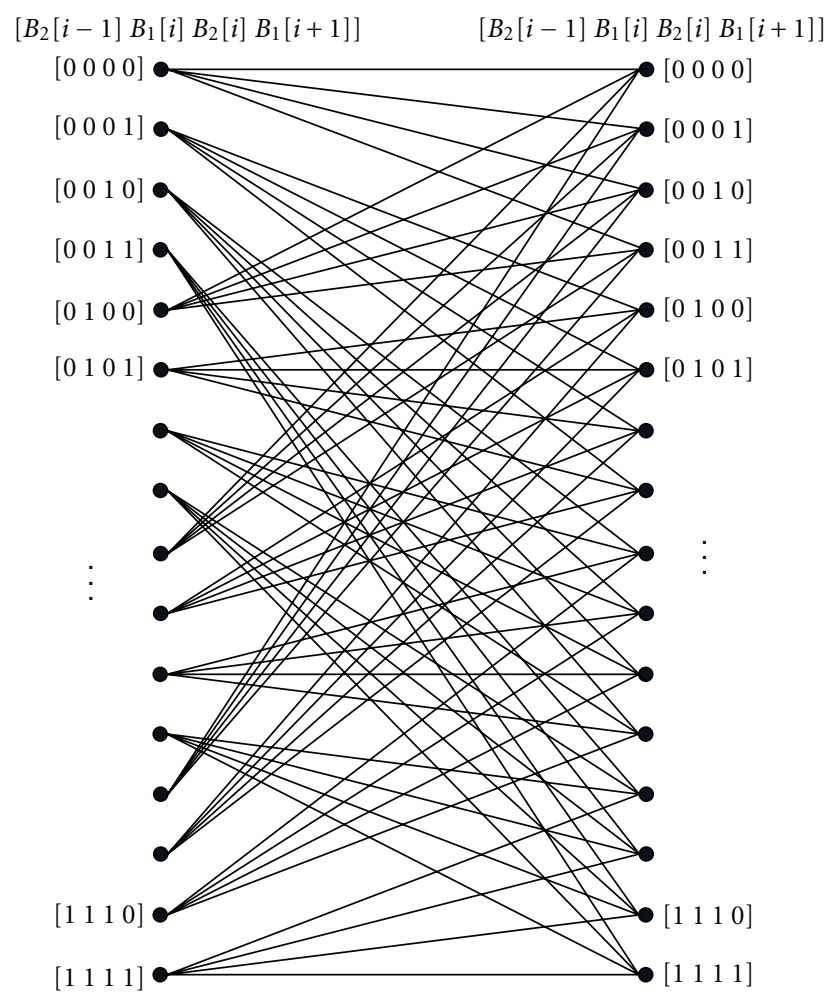

Figure 7: Trellis connectivity.

5.3. Viterbi Algorithm-Based Receivers. Since ISI is inherently present in the proposed offset system, the optimal receiver is the maximum likelihood sequence detector (MLSD). The Viterbi algorithm [25] is a very well known algorithm for implementing the MLSD in a computationally tractable manner. As shown in [26] and implied by [25, Section 2], the usual implementation of the Viterbi algorithm yields the MLSD only if the noise is memoryless and is independent from sample to sample. In our case, however, this is not true as the noise has temporal correlation as indicated by (10).

In order to reduce the impact of the temporal noise correlation, we carried out noise whitening over different observation windows. that is, the Viterbi algorithm was run not on the received samples, but on $\mathbf{R n n}^{-1 / 2} \mathbf{y}[i]$, where $\mathbf{R n n}$ denotes the covariance of the noise vector and $\mathbf{y}[i]$ denotes the received vector as given by (4) for the one shot case and by (23) for the windowed case. Although this method whitens the noise locally, it does not whiten the noise over the entire received burst and thus is an approximation to the ML solution.

5.3.1. Rectangular Pulse. A cursory examination of (4) reveals a channel memory of 3 symbol times and with BPSK signaling with 2 transmit antenna this leads to a total of $\left(2^{2}\right)^{3}=64$ states in the trellis. However, a more careful inspection using the structure of matrices $P$ and $R$ from (2), indicates that the channel memory can be reduced to 4 bits and thus results in 16 states as shown in Figure 7.

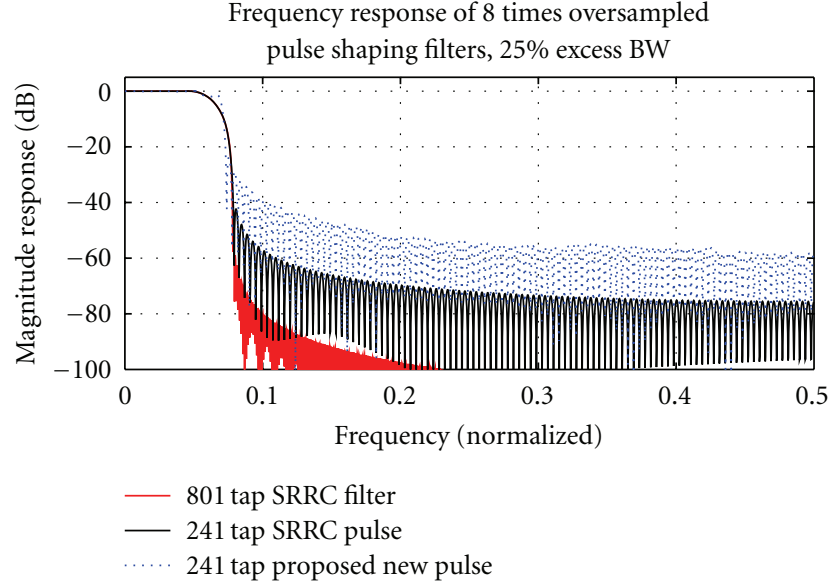

FiguRE 8: Frequency response of proposed new pulse compared with SRRC Filter.

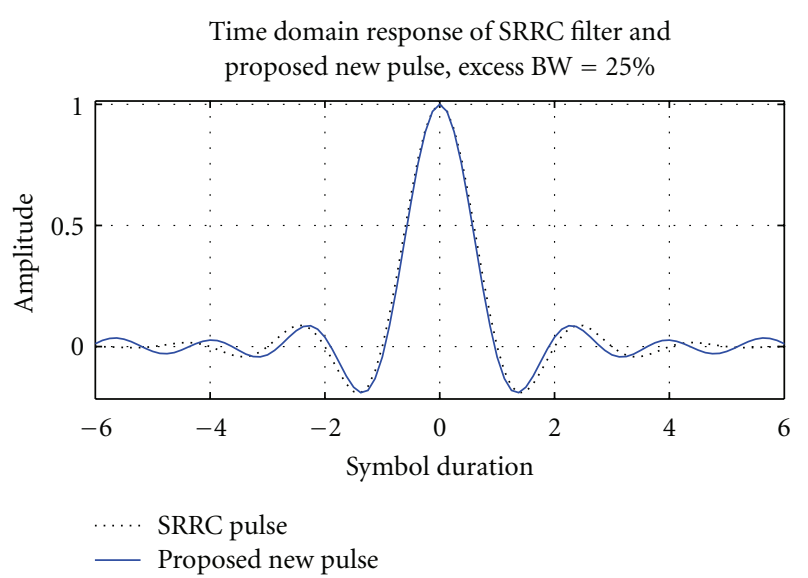

FIgURE 9: Time response of proposed new pulse compared with SRRC Filter.

5.3.2. Raised Cosine Pulse. When the SRRC pulse shape is employed the channel memory depends on the length of the filters employed. Our simulations employed a SRRC filter of length 21 symbols with $25 \%$ excess bandwidth, and thus the ISI extends over 20 symbol durations. This causes the trellis to grow unacceptably large for implementation purposes. The optimal trellis for a pulse with $L$ symbol ISI and for a system using $M_{T}$ transmitters and an $M$-ary constellation is $\left(M^{M_{T}}\right)^{L}$ long. This is usually impractical to implement and so suboptimal trellis decoders are often employed. In our simulations, we have opted for a suboptimal solution that uses a very similar 16 state trellis as is used for the rectangular pulse and pretends that the ISI is only from the adjacent symbols and ignores the ISI from the other interfering symbols. This is clearly suboptimal. However, since most of the interference power comes from the adjacent symbols, this suboptimal receiver captures most of the performance gain and the improvements by going to more complex receivers are likely to be marginal. In passing, we note that the conventional scheme does not have ISI and so sequence detection does not improve its performance. 
The 16 state Viterbi trellis used for the sequence detection receivers is shown in Figure 7.

\section{Pulse Shape Design for MIMO with Timing Offset}

In this section, we propose robustness to IAI (defined in (1)) as a new criterion for pulse shape design. The key idea is the following: once the transmitters are offset from each other, the IAI is controlled by the correlation of the transmit pulse shape with the received pulse shape at an offset equal to the offset of the symbol boundaries. Without an offset, this criterion is no longer valid since the IAI is given by the correlation of the two pulses at zero offset (which is unity for all normalized pulse shapes). Similar to the formulation of (3) in [18], we minimize the cost function

$$
\xi=\xi_{s}+\sum_{n \in S_{\mathrm{ISI}}} \gamma(g[n]-d[n])^{2}+\sum_{n \in S_{\mathrm{IAI}}} \eta g^{2}[n],
$$

where $\xi_{s}$ is the stop band energy of the square root Nyquist (M) discrete-time filter given by $h[n]$ which runs at $M$ samples/symbol, where $n$ is the discrete time index. $d[n]$ is the response of the convolution of the two square root Nyquist filters being designed with the target response given by $g[n]$. $S_{\text {ISI }}$ and $S_{\text {IAI }}$, respectively, identify different subsets of samples of $n$ as shown below. $\gamma$ and $\eta$ are weighting functions that allow us to trade off one constraint with another. In an ideal square root Nyquist filter, $g[n]=h[n] * h[-n]$, where $*$ denotes convolution and $g[n]$ satisfies the no-ISI Nyquist criterion given by

$$
g[n]= \begin{cases}1, & \text { if } n=0, \\ 0, & \text { if } n=m M, m \neq 0 \\ \text { arbitrary, } & \text { if } n \neq m M .\end{cases}
$$

Thus, $S_{\text {ISI }}=\{0, \pm M, \pm 2 M, \ldots\}$ is the subset of $n$, where constraints are placed to minimize the ISI.

In order to reduce the IAI, we need to lower the energy of $g[n]$ at the offset points. Thus, for example, for an offset of $T / 2$, the sum of the square of the samples of $g[n]$ at $\pm M / 2, \pm M(1+1 / 2), \pm M(2+1 / 2)$, and so on need to be lowered. By choosing $S_{\text {IAI }}$ to be the set $\{ \pm M / 2, \pm M(1+$ $1 / 2), \pm M(2+1 / 2), \ldots\}$ and by choosing appropriate weights, $\gamma$ and $\eta$, we can perform a tradeoff between the reduction of ISI and IAI. In [18], an iterative method for designing a filter conforming to such a cost function is described in detail and is used by us.

Using this method of pulse shape generation, we can create a family of pulses that have various tradeoffs of ISI, IAI and stop-band attenuation. Here, we show an example of such a pulse, by choosing an excess bandwidth of $25 \%$ and $\gamma=1$ and $\eta=0.6$. The key properties of this pulse in comparison to the square root raised cosine pulse shape are summarized in Table 2 .

It may be seen that the residual ISI goes up from $-74 \mathrm{~dB}$ (practically zero) in the case of two SRRC pulses convolved with each other to $-19 \mathrm{~dB}$ (still pretty low) in the case of
TABLE 2: Square root raised cosine versus new pulse.

\begin{tabular}{lcc}
\hline & SRRC $*$ SRRC & New pulse $*$ new pulse \\
\hline Residual ISI $(\mathrm{dB})$ & -74 & -19 \\
$T / 2$ IAI $(\mathrm{dB})$ & -0.58 & -1.02 \\
\hline
\end{tabular}

the two proposed pulses convolved with each other. The IAI power caused by an offset of half a symbol time $(T / 2)$, however, has been improved from about $-0.58 \mathrm{~dB}$ to about $-1.02 \mathrm{~dB}$.

The frequency response of 3 different filters are plotted in Figure 8. It may be seen that compared to the frequency response of a SRRC filter of same length, the proposed pulse has worse stop band attenuation. The peak sidelobe level is still close to $-30 \mathrm{~dB}$ below the main lobe and is thus considered acceptable. The time domain response is shown in Figure 9, where it may be seen that the two pulse shapes are similar though ISI has increased for the proposed pulse at the benefit of a lower IAI at T/2 offset.

Although we are showing only a single pulse shape here, different designers could come up with different pulse shapes depending on different weights imposed in (27) depending on various system parameters. Our emphasis here is on the importance of minimization of IAI as a filter design parameter for offset MIMO systems not so much on the exact choice of the parameters which might vary from system to system.

\section{Simulation Results}

The simulations have been done as a set of experiments where, in each case, comparisons have been made to similar aligned systems. In all cases, the channel is assumed to be known perfectly at the receiver. Each simulation also assumes a block fading model, where the channel is independent from block to block and is assumed to be constant over the duration of each block. The channel coefficients have been generated as samples from a mean zero, unit variance complex Gaussian random variable. To obtain statistically reliable results, each datapoint is obtained by simulating at least 10000 blocks. The total transmit power is held constant irrespective of the number of transmitters by normalizing the output power from each transmitter by the number of transmitters, $M_{T}$. The performance metric of choice is symbol error rate (SER) or bit error rate (BER) which is plotted in the following graphs as a function of $E_{s} / N_{0}$, the ratio of the symbol energy $\left(E_{s}\right)$ to the noise power spectral density $\left(N_{0}\right)$. The performance is compared at a SER equal to $10^{-2}$.

7.1. Comparison with OSIC VBLAST. In Figures 10 and 11 , the performance of the proposed system with MMSE receivers is compared to that of a traditional aligned VBLAST with ordered successive interference cancellation (OSIC). A $2 \mathrm{Tx}-2 \mathrm{Rx}$ system with quadrature phase shift keying (QPSK) modulation is simulated with blocks containing 128 symbols. The performance of systems with rectangular pulse shaping is shown in Figure 10 and that of systems with raised 


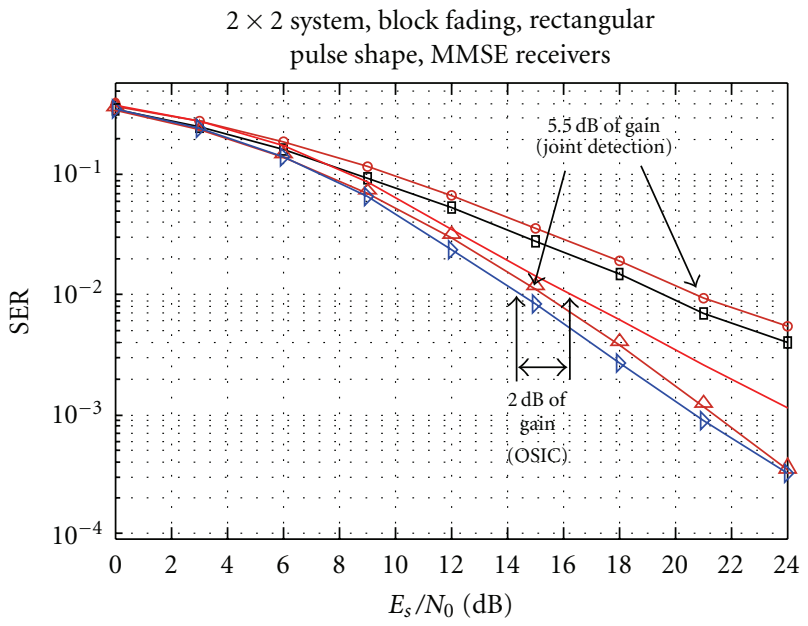

$\rightarrow$ Offset MIMO: MMSE one shot joint detection

$\triangle$ Offset MIMO: MMSE 2 adjacent symbols window joint detection

$\rightarrow$ Baseline: MMSE joint detection

— Baseline: MMSE OSIC

$\rightarrow$ Offset MIMO: MMSE one shot OSIC

FIGURE 10: Offset MIMO with MMSE Rx compared OSIC VBLAST, $\left(M_{T}, M_{R}\right)=(2,2)$, modulation $=$ QPSK.

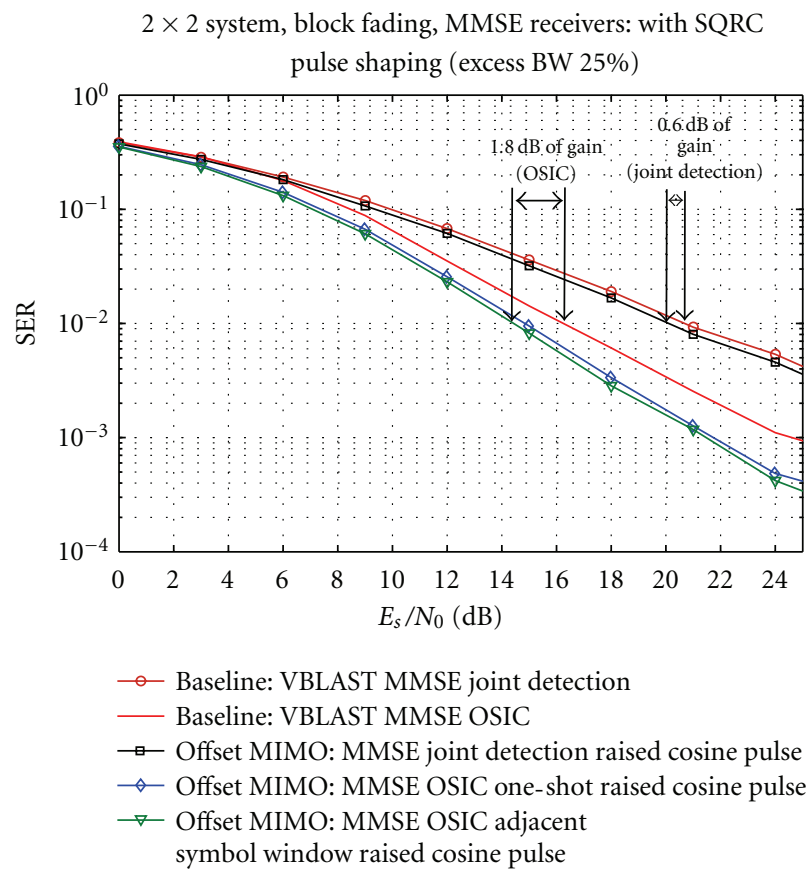

FIgURE 11: Offset MIMO with SRRC pulse shaping versuss OSIC VBLAST, $\left(M_{T}, M_{R}\right)=(2,2)$, modulation = QPSK.

cosine pulse shaping with $25 \%$ excess bandwidth is shown in Figure 11. The square root raised cosine (SRRC) filters on the transmitter and receiver sides have both been truncated to 13 symbols.

In either case, the comparison has been made to the "best" aligned VBLAST scheme which is when the VBLAST receivers employ OSIC [23]. It may be seen that the

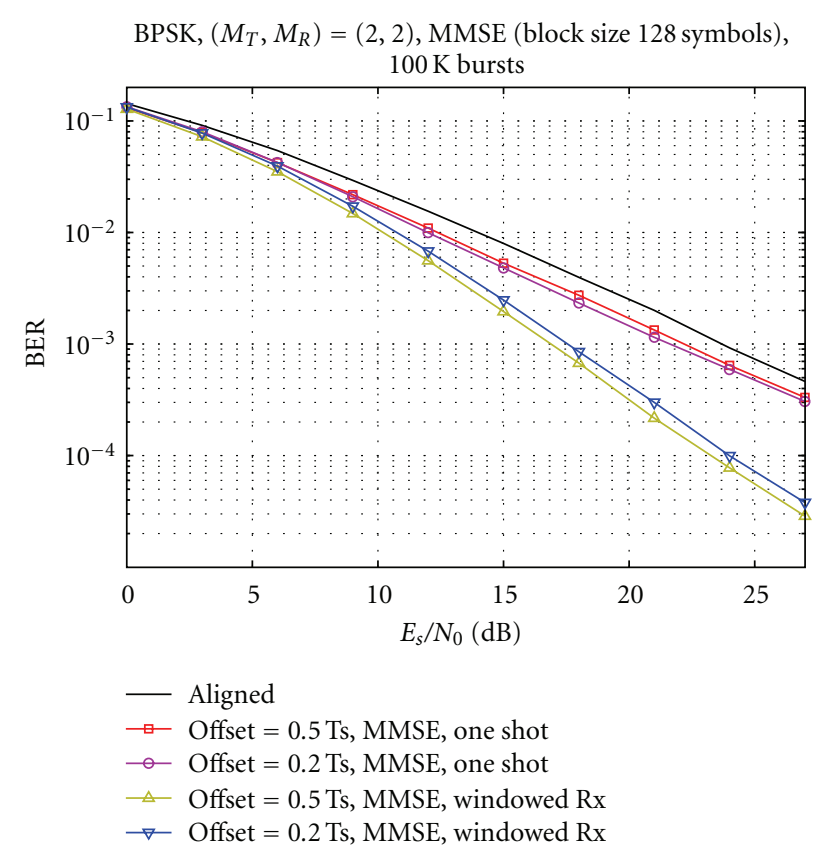

FIGURE 12: BER Performance for BPSK with various offsets, $\left(M_{T}, M_{R}\right)=(2,2)$.

proposed system outperforms the VBLAST scheme both when rectangular pulse shaping is employed as well as when the raised cosine pulse shape is employed. In the latter, and more practical case, the gain is about $1.8 \mathrm{~dB}$ (at a BER of $10^{-2}$ ) when OSIC is employed on both the proposed system as well as on aligned traditional VBLAST.

7.2. Performance for Various Offsets. In this set of simulations, Figure 12 shows the performance of a $2 \times 2$ system with BPSK modulation for various offsets between the first and second transmitters. A rectangular pulse shape is used. The performance of both an one-shot as well as a windowed receiver is shown. It may be seen that the MMSE windowed receiver achieves a lower BER with offset of $0.5 \mathrm{~T}$, whereas when the one-shot receiver is employed, an offset of $0.2 \mathrm{~T}$ is better at higher SNRs. More details on the performance at various offsets as well as an analytical derivation of an optimal offset for a $\left(M_{T}, M_{R}\right)=(2,1)$ may be found in our prior work [21].

7.3. Performance of Sequence Detection-Based Receivers. In Figure 13, the performance of Viterbi algorithm-based receivers are shown in comparison to that for a traditional $2 \times 2$ MIMO system employing symbol-by-symbol ML detection. BPSK modulation with rectangular pulse shaping was used in a $\left(M_{T}, M_{R}\right)=(2,2)$ system. Three curves are shown for offset MIMO: (i) without employing noise whitening, (ii) using noise whitening on a one shot case ( $W=0$ ), and (iii) using noise whitening on an extended window basis $(W=2)$.

It may be seen that without noise whitening, the performance of the Viterbi algorithm-based receiver is 
TABLE 3: Timing aligned MIMO compared to timing offset MIMO.

\begin{tabular}{lll}
\hline & Offset MIMO & VBLAST \\
\hline Matched filter rate & $M_{T}$ samp/symb & 1 samp/symb \\
& Needs inverse (or Pseudoinverse) of $S M_{T} \times S M_{T}$ matrix & Needs inverse (or Pseudoinverse) of $M_{R} \times M_{T}$ matrix \\
& Performance gain $\sim 5 \mathrm{~dB}$ & \\
One-shot MMSE & $\begin{array}{l}\text { Needs Inverse (or Pseudoinverse) of } M_{T} M_{R} \times M_{T} M_{R} \\
\text { matrix }\end{array}$ & Needs inverse (or Pseudoinverse) of $M_{R} \times M_{T}$ matrix \\
& $\begin{array}{l}\text { Performance gain } \sim 1.5 \mathrm{~dB} \\
\text { More gains from more complexity Complexity grows }\end{array}$ & \\
Windowed MMSE & Nith window size & \\
& $\begin{array}{l}\text { Performance gain } \sim 6 \mathrm{~dB} \\
\text { Trellis size (and thus complexity) can be traded for } \\
\text { Trellis based receivers }\end{array}$ & \\
& performance & Symbol-by-symbol ML receivers are optimal \\
Performance gain $\sim 0.5 \mathrm{~dB}$ & \\
New pulse shapes & $\begin{array}{l}\text { Gains from new pulses that lower IAI } \\
\text { Performance gain } \sim 1 \mathrm{~dB}\end{array}$ & No gains from new pulse shapes \\
\hline
\end{tabular}

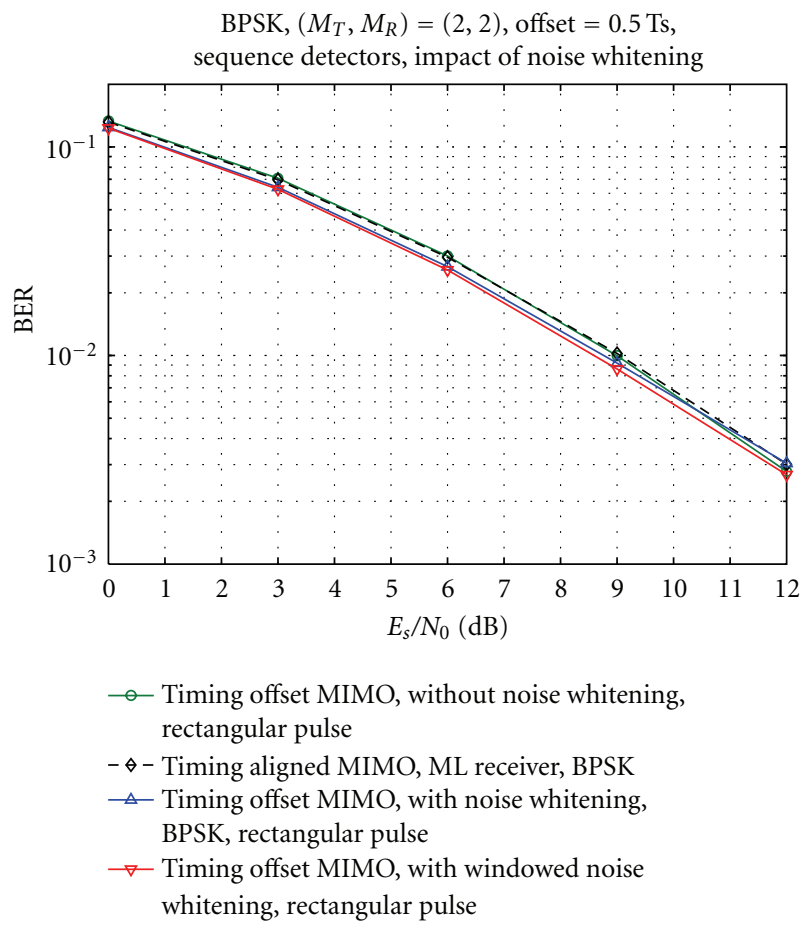

Figure 13: Impact of noise whitening on trellis-based receivers, $\left(M_{T}, M_{R}\right)=(2,2)$, modulation $=$ BPSK.

approximately equal to that of the traditional symbol aligned system with ML detection. However, when noise whitening is employed, we pick up a gain of about $0.5 \mathrm{~dB}$ at a BER of $10^{-2}$. While the gains in this case are admittedly smaller, in some systems even a $0.5 \mathrm{~dB}$ gain in performance might be worth the additional complexity.

7.4. Performance of a $3 \times 3$ System. In Figure 14, we present the results of a $3 \times 3$ MIMO system with offset transmission with MMSE joint detection receivers. In this case, there are two offsets, and they have been set to $T / 3$ and $2 T / 3$. It may be

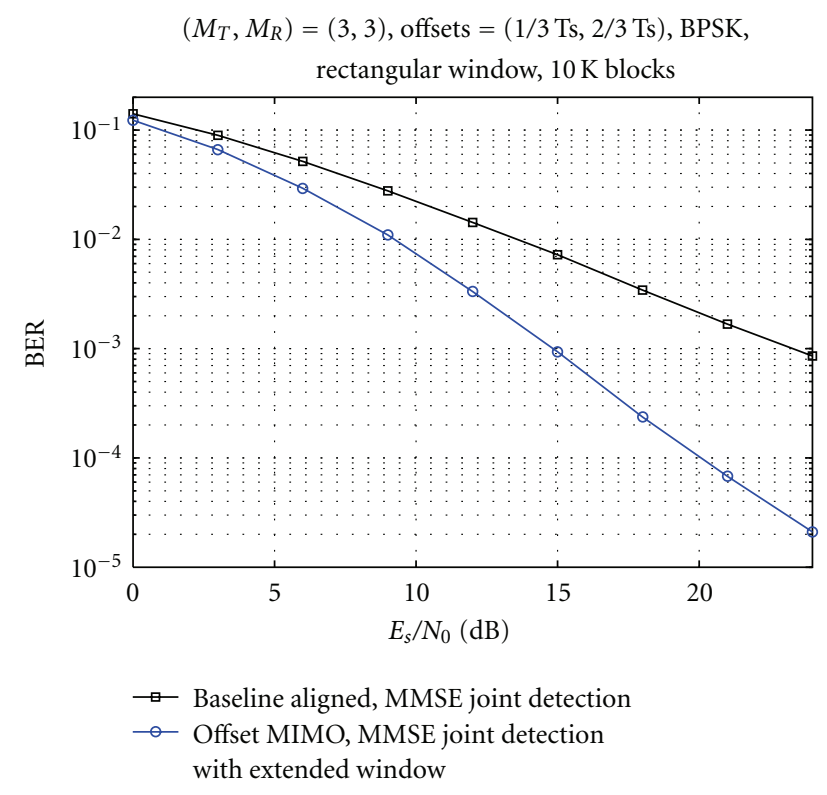

Figure 14: Performance of a $3 \times 3$ system, $\left(M_{T}, M_{R}\right)=(3,3)$, modulation $=$ BPSK.

seen that the performance gains are over $6 \mathrm{~dB}$ (when SER = $10^{-2}$ ) when used with a rectangular pulse shape.

7.5. ZF Receivers. The performance of the optimal ZF receiver is plotted against the performance of the $Z F$ receiver presented by Shao et al. in Figure 15. It may be seen that while the Shao et al. receiver degrades significantly with increasing block size $S$, the optimal ZF receiver has a very weak dependence on block size. In Figure 15, the $x$-axis has been plotted in terms of $E t / N_{o}=\left(\left(S T+\tau_{1}\right) / S T\right) E_{s} / N_{0}$, where $S$ is the block size, $T$ the symbol duration and $\tau_{1}$ the offset. As shown in [8], this ensures that the data rate across all the systems is the same. We emphasize, however, that normalizing the data rate does not imply that all the 


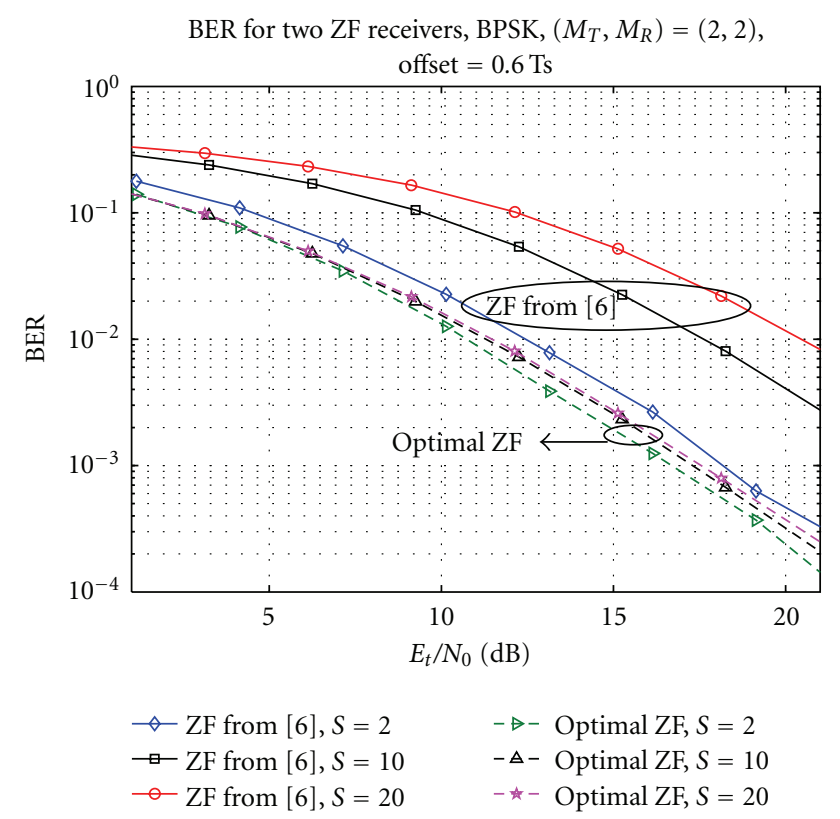

FIGURE 15: Optimal ZF receiver versus ZF receiver from [8].

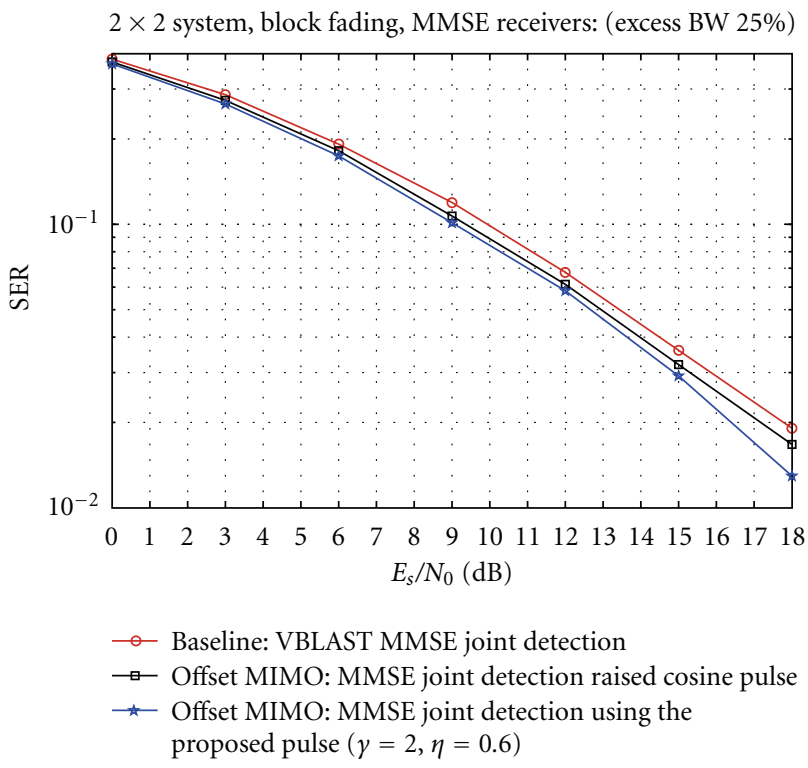

FIgURe 16: Performance of new pulse shaping.

block sizes are equally efficient. Very short block sizes lead to considerably less spectral efficiency due to the inter gap idle time representing a higher overhead.

7.6. Performance of New Pulse Shaping. To show the benefits of the proposed pulse shaping, the performance of a system using a member of the new proposed pulse family is compared in Figure 16 to the performance of a system using an SRRC pulse. Both systems were simulated using an MMSE joint detection receiver. It may be seen that the performance is improved by using the new pulse. Note that this, approximately $0.25 \mathrm{~dB}$ additional, improvement comes with absolutely no additional system complexity and can thus be regarded of as "free". Although not shown, the new pulse could be used with the trellis based receivers or zero forcing receivers as well.

\section{Conclusions}

A novel MIMO transmission scheme, using transmitters that are intentionally offset in time from each other, has been analyzed in this paper. A nonzero (but known) symbol timing offset is introduced between the signals transmitted from the different transmitters to take advantage of the inefficiencies in practical signalling systems. It is shown that a suitably designed receiver can utilize this information to extract significant performance gains. This transmission scheme is studied in conjunction with different kinds of receivers: ZF, MMSE receivers, as well as MIMO MMSE receivers with ordered successive interference cancellation and trellis-based sequence detection-based receivers.

A new pulse shape design that lowers IAI has also been introduced and is shown to increase the gains of such offset transmission schemes.

A summary of highlights of the comparison between an aligned scheme like VBLAST with the proposed scheme is shown in Table 3. The main source of complexity increase is shown along with the performance gain. The performance gain is shown for a $\left(M_{T}, M_{R}\right)=(2,2)$ system with an offset of $T / 2$ using BPSK at a BER of $2 \times 10^{-3}$ in comparison to an aligned system.

\section{References}

[1] P. W. Wolniansky, G. J. Foschini, G. D. Golden, and R. A. Valenzuela, "V-BLAST: an architecture for realizing very high data rates over the rich-scattering wireless channel," in Proceedings of the International Symposium on Signals, Systems and Electronics (ISSSE'98), pp. 295-300, 1998.

[2] J. Proakis, Digital Communications, McGraw-Hill Science, 2000.

[3] ETSI, "Digital Video Broadcasting (DVB), ETSI EN 302307 V1.1.2 (2006-06)," 2006.

[4] C. T. L. Inc, "Data-over-cable service interface specifications docsis 2.0, radio frequency interface specification, $\mathrm{cm}$ sprfiv2.0- i11-060602".

[5] C. Tepedelenlioglu and R. Challagulla, "Low-complexity multipath diversity through fractional sampling in OFDM," IEEE Transactions on Signal Processing, vol. 52, no. 11, pp. 31043116, 2004.

[6] B. D. Rao and A. Das, "Multiple antenna enhancements via symbol timing relative offsets (MAESTRO)," in Proceedings of the 18th Annual IEEE International Symposium on Personal, Indoor and Mobile Radio Communications (PIMRC '07), pp. 1-5, September 2007.

[7] S. Shao, Y. Tang, J. Liang, X. Li, and S. Li, "A modified VBLAST system for performance improvement through introducing different delay offsets to each spatially multiplexed data streams," in Proceedings of the IEEE Wireless Communications and Networking Conference (WCNC '07), pp. 1062-1067, IEEE, 2007. 
[8] S. Shao, Y. Tang, T. Kong, K. Deng, and Y. Shen, "Performance analysis of a modified V-BLAST system with delay offsets using zero-forcing detection," IEEE Transactions on Vehicular Technology, vol. 56, no. 6, pp. 3827-3837, 2007.

[9] Q. Wang, Y. Chang, and D. Yang, "Deliberately designed asynchronous transmission scheme for MIMO systems," IEEE Signal Processing Letters, vol. 14, no. 12, pp. 920-923, 2007.

[10] K. Barman and O. Dabeer, "Improving capacity in MIMO systems with asynchronous PAM," in Proceedings of the International Symposium on Information Theory and its Applications (ISITA '08), pp. 1-6, December 2008.

[11] A. Wittneben, "New bandwidth efficient transmit antenna modulation diversity scheme for linear digital modulation," in Proceedings of the IEEE International Conference on Communications (ICC '93), vol. 3, pp. 1630-1634, Geneva, Switzerland, 1993.

[12] J. Tan and G. L. Stuber, "Multicarrier delay diversity modulation for MIMO systems," IEEE Transactions on Wireless Communications, vol. 3, no. 5, pp. 1756-1763, 2004.

[13] A. Dammann, S. Plass, and S. Sand, "Cyclic delay diversitya simple, flexible and effective multi-antenna technology for OFDM," in Proceedings of the IEEE 10th International Symposium on Spread Spectrum Techniques and Applications (ISSSTA '08), pp. 550-554, August 2008.

[14] F. J. Harris, Multirate Signal Processing for Communication Systems, Prentice Hall PTR, 2004.

[15] N. C. Beaulieu, C. C. Tan, and M. O. Damen, "A "better than" Nyquist pulse," IEEE Communications Letters, vol. 5, no. 9, pp. 367-368, 2001.

[16] S. S. Mneina and G. O. Martens, "Maximally flat delay Nyquist pulse design," IEEE Transactions on Circuits and Systems II, vol. 51, no. 6, pp. 294-298, 2004.

[17] J. K. Liang, R. J. P. deFigueiredo, and F. C. Lu, "Design of optimal Nyquist, partial response, Nth band, and nonuniform tap spacing FIR digital filters using linear programming techniques," IEEE Transactions on Circuits and Systems, vol. 32, no. 4, pp. 386-392, 1985.

[18] B. Farhang-Boroujeny, "A square-root Nyquist (M) filter design for digital communication systems," IEEE Transactions on Signal Processing, vol. 56, no. 5, pp. 2127-2132, 2008.

[19] N. C. Beaulieu and M. O. Damen, "Parametric construction of Nyquist-I pulses," IEEE Transactions on Communications, vol. 52, no. 12, pp. 2134-2142, 2004.

[20] S. Verdu, Multiuser Detection, Cambridge University Press, 1998.

[21] A. Das and B. D. Rao, "Impact of receiver structure and timing offset on MIMO spatial multiplexing," in Proceedings of the IEEE 9th Workshop on Signal Processing Advances in Wireless Communications (SPAWC'08), pp. 466-470, July 2008.

[22] S. M. Kay, Fundamentals of Statistical Signal Processing, Prentice Hall, 1993.

[23] A. Paulraj, R. Nabar, and D. Gore, Introduction to Space-Time Wireless Communications, Cambridge University Press, 2003.

[24] R. Bohnke, D. Wubben, V. Kuhn, and K. D. Kammeyer, "Reduced complexity MMSE detection for BLAST architectures," in Proceedings of the IEEE Global Telecommunications Conference (GLOBECOM '03), vol. 4, pp. 2258-2262, December 2003.

[25] G. D. Forney, "The viterbi algorithm," Proceedings of the IEEE, vol. 61, no. 3, pp. 268-278, 1973.

[26] A. Kavcic and J. M. F. Moura, "The Viterbi algorithm and Markov noise memory," IEEE Transactions on Information Theory, vol. 46, no. 1, pp. 291-301, 2000. 